\title{
Canagliflozin: A Review in Type 2 Diabetes
}

\author{
Emma D. Deeks ${ }^{1}$ André Scheen
}

(c) Springer International Publishing Switzerland 2017

\begin{abstract}
Canagliflozin $\left(\operatorname{Invokana}^{\circledR}\right.$ ) is a sodium-glucose co-transporter-2 (SGLT2) inhibitor indicated in various countries worldwide for the once-daily oral treatment of type 2 diabetes (T2D). Unlike many T2D therapies, canagliflozin lowers blood glucose levels independently of insulin, with the inhibition of SGLT2 reducing renal reabsorption of glucose and increasing excretion of glucose in the urine. In well-designed clinical trials, canagliflozin (as first-line monotherapy or add-on therapy to other antihyperglycaemic agents) improved glycaemic control in adults with T2D, including those of older age and/or at high cardiovascular (CV) risk, and also had beneficial effects on their bodyweight and BP; whether the drug impacts the CV risk profile of T2D is currently being evaluated in the large CV outcomes study CANVAS. Canagliflozin was generally well tolerated, had a low risk of hypoglycaemia and was most commonly associated with adverse events such as genital and urinary tract infections and increased urination, consistent with its mechanism of action. Thus, canagliflozin is a useful monotherapy and add-on therapy option for the management of T2D in adults, and its CV profile is eagerly awaited.
\end{abstract}

The manuscript was reviewed by: ((to come))

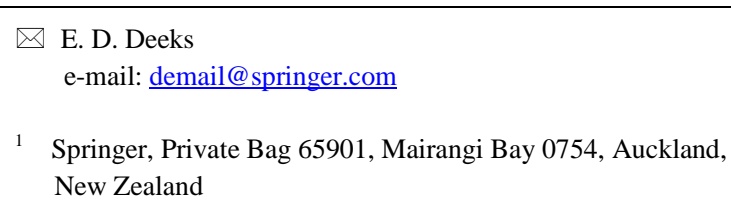

\section{Canagliflozin: clinical considerations in type 2} diabetes

Lowers blood glucose levels by increasing urinary glucose excretion, an effect independent of insulin Provides effective glycaemic control as first-line monotherapy or as add-on therapy

Associated with reductions in bodyweight and BP Generally well tolerated with a low risk of hypoglycaemia 


\section{Introduction}

Type 2 diabetes (T2D) is a chronic progressive metabolic disease characterized by hyperglycaemia, due to insulin resistance/insufficient insulin production [1]. Patients are often obese, have lipid disturbances and elevated BP, and are at an increased risk of microvascular and macrovascular complications [1]. Achieving good glycaemic control [e.g. $\mathrm{HbA}_{1 \mathrm{C}}$ level $<7 \%$ ] is a key management goal, for which numerous antihyperglycaemic agents (AHAs) with varying mechanisms of action are now available [2]. Sodium-glucose co-transporter-2 (SGLT2) inhibitors are a relatively recent AHA class. By inhibiting SGLT2 (a key protein in glucose resorption in the kidney), these drugs increase urinary glucose excretion (UGE), causing blood glucose levels to decline independently of insulin. One of the most widely available SGLT2 inhibitors is canagliflozin $\left(\right.$ Invokana $^{\circledR}$ ), which is approved for the treatment of T2D in various countries worldwide, including the USA and EU. This article reviews data relevant to the use of canagliflozin in T2D in the EU; fixed-dose canagliflozin/metformin tablets are also now available but are beyond the scope of this article.

\section{Pharmacodynamic Properties}

Canagliflozin is a potent competitive inhibitor of SGLT2 [3, 4], and thus reduces both renal glucose reabsorption and the renal threshold for glucose $\left(\mathrm{RT}_{\mathrm{G}}\right)$, with subsequent increases in UGE [5-7]. These increases in UGE reduce plasma glucose levels [5-7], provide calorie and thus bodyweight loss (Sect. 4.2) and have an osmotic diuretic effect (that may reduce BP; Sect. 4.3) [7], although transient early increases in urine output with canagliflozin may also reflect increased natriuresis [8]. Maximal $\mathrm{RT}_{\mathrm{G}}$ suppression in $\mathrm{T} 2 \mathrm{D}$ patients was seen with canagliflozin $300 \mathrm{mg} / \mathrm{day}$ in phase 1 trials, with $24-\mathrm{h}$ mean $\mathrm{RT}_{\mathrm{G}}$ values reduced from $\approx 13$ to $4-5 \mathrm{mmol} / \mathrm{L}$ (i.e. values greater than plasma glucose levels usually associated with hypoglycaemia symptoms, indicating a low hypoglycaemia risk; Sect. 5.3) [7].

Canagliflozin is highly selective for SGLT2 in vitro [ $\underline{3}, \underline{4}]$, with one study, for instance, demonstrating $\approx 200$-fold greater affinity of the drug for SGLT2 than SGLT1 [3]]. However, as SGLT1 is key in gastrointestinal glucose absorption, canagliflozin in the small intestines after oral administration may transiently inhibit intestinal SGLT1, and thus glucose absorption [9]. Indeed, in small placebo-controlled crossover studies in T2D patients [10] or healthy volunteers [11], canagliflozin reduced postprandial glucose (PPG) excursions by both non-renal (possibly via intestinal SGLT1 inhibition) and renal (via SGLT2 inhibition) mechanisms. Notably, canagliflozin $300 \mathrm{mg} / \mathrm{day}$ was associated with less extensive PPG excursions, lower 24-h mean $\mathrm{RT}_{\mathrm{G}}$ and greater 24-h UGE than dapagliflozin $10 \mathrm{mg} / \mathrm{day}$ in healthy volunteers [12]. Various measures of $\beta$-cell function improved with canagliflozin regimens in T2D trials $[\underline{7}, \underline{13-}$ 17], likely as an indirect consequence of reduced glucotoxicity.

Canagliflozin (100 or $300 \mathrm{mg} /$ day) was generally associated with small changes in serum electrolytes (including sodium, calcium, bicarbonate, phosphate, potassium and magnesium) [18], normalization of serum magnesium levels in hypomagnesaemia [19], and reductions in serum uric acid levels (possibly via increased urinary uric acid excretion) [20] in pooled (post hoc $[19,20]$ ) analyses of phase 3 trials in T2D patients. The most common serum electrolyte abnormality of interest with canagliflozin 100 or $300 \mathrm{mg}$ /day was potassium above the upper limit of normal plus $>15 \%$ increase from baseline, both in patients with an estimated glomerular filtration rate (eGFR) $\geq 60 \mathrm{~mL} / \mathrm{min} / 1.73 \mathrm{~m}^{2}(5$ and 7 vs. 5\% of placebo recipients) and in those with an eGFR $\geq 45$ to $<60 \mathrm{~mL} / \mathrm{min} / 1.73 \mathrm{~m}^{2}$ (5 and 9 vs. $6 \%$ ) [18].

Elderly patients with T2D who added canagliflozin to their current AHA regimen in a phase 3 study (Sect. 4.4.2) had small but significant reductions in BMD at the total hip (but not other skeletal sites) over 104 weeks of therapy compared with adding placebo, with increases in biomarkers of bone turnover also seen over 52 weeks in the canagliflozin versus placebo groups; these changes were partly due to bodyweight loss, and bone strength was not impacted [21]. 


\section{Pharmacokinetic Properties}

Canagliflozin is rapidly absorbed after oral administration, reaching maximum plasma concentrations $1-2 \mathrm{~h}$ post-dose in T2D patients [5] and healthy volunteers [7]. The drug has a mean absolute oral bioavailability of $\approx 65 \%$ [22] and reaches steady-state in 4-5 days [ [, 7]. Exposure increases in proportion to dose and there is up to $36 \%$ accumulation of the drug in plasma at the recommended dosages (100 or $300 \mathrm{mg} /$ day) [5, 7]. Food does not impact canagliflozin pharmacokinetics [23], enabling it to be taken with or without food [7]; however, as canagliflozin may help reduce PPG excursions by delaying glucose absorption in the intestine (Sect. 2), administration before the first meal of the day is advised [7].

Canagliflozin is extensively (99\%) plasma protein bound, and has a mean volume of distribution at steady state of 83.5 L after intravenous infusion [7]. Metabolism of canagliflozin occurs primarily via $O$-glucuronidation (by UGT1A9 and UGT2B4), producing two main inactive metabolites [24]; metabolism via CYP3A4 is minimal $(\approx 7 \%)$ [7]. Canagliflozin is eliminated via the faeces $(41.5 \%$ as parent drug; $10.2 \%$ as metabolites) and urine (33\%, mainly $O$ glucuronide metabolites), and recommended doses have renal clearance rates of $1.30-1.55 \mathrm{~mL} / \mathrm{min}$ [7]. The mean elimination half-life of canagliflozin 100 or $300 \mathrm{mg} /$ day in T2D patients was 13.7 and $14.9 \mathrm{~h} \mathrm{[5]}$.

Mild or moderate hepatic impairment does not alter canagliflozin pharmacokinetics to a clinically relevant extent [25] and does not necessitate dosage adjustment [7]; the drug has not been assessed, and is thus not recommended, in severe hepatic impairment [7]. Renal impairment increases canagliflozin exposure and reduces pharmacodynamic response to the drug [25]. The canagliflozin dosage does not require adjustment in patients with an eGFR of 60 to $<90$ $\mathrm{mL} / \mathrm{min} / 1.73 \mathrm{~m}^{2}$; however, if eGFR persistently declines below 60 or $45 \mathrm{~mL} / \mathrm{min} / 1.73 \mathrm{~m}^{2}$, dosage adjustment/consideration or discontinuation is necessary [7]. Canagliflozin should not be initiated in patients with an eGFR $<60 \mathrm{~mL} / \mathrm{min} / 1.73 \mathrm{~m}^{2}$ or used in patients with end-stage renal disease/on dialysis. Renal function monitoring is advised [7].

Higher than therapeutic concentrations of canagliflozin did not inhibit or induce key CYP isoenzymes in vitro, and there was no clinically relevant impact of canagliflozin on CYP3A4 in vivo [7]. However, as canagliflozin is metabolized by UGT1A9 and UGT2B4, and transported by p-gp and BCRP, drugs that induce these enzymes may reduce canagliflozin exposure; thus, if coadministered, canagliflozin dosage adjustment (and, in some instances, additional AHAs) may be necessary [7]. There is also potential for canagliflozin exposure to be reduced by cholestyramine, necessitating staggered administration [7]. Canagliflozin weakly inhibits p-gp and may thus increase plasma concentrations of p-gp substrates; monitoring is advised [ $\underline{5}, \underline{7}]$. Intestinal BCRP inhibition by canagliflozin cannot be ruled out and may increase exposure to drugs transported by BCRP [7]. Canagliflozin may augment the effects of diuretics, increasing dehydration and hypotension (Sect. 5.2) risks [7]. There is also an increased risk of hypoglycaemia if canagliflozin is used in combination with an insulin secretagogue or insulin (Sect. $\underline{5.3}$ ); reduction of the insulin or insulin secretagogue dosage may be required [7].

\section{Therapeutic Efficacy}

The clinical efficacy of oral canagliflozin, as monotherapy or add-on therapy, in adults with inadequately-controlled T2D, has been evaluated in numerous placebo- and/or active comparator-controlled trials of randomized, double-blind (or open-label [26]) design and 16-52-weeks' duration; some studies also had 26- to 78-week double-blind extensions. Unless otherwise specified, trials were phase 3 and used the change from baseline in $\mathrm{HbA}_{1 \mathrm{C}}$ (usually at 26 or 52 weeks; range 16-52 weeks) as the primary endpoint. Real-world data are also now available. Discussion focuses on recommended canagliflozin dosages (i.e. 100 or $300 \mathrm{mg} /$ day); some data are from abstracts [27-32]. 


\subsection{Glycaemic Parameters}

In patients with T2D inadequately controlled by diet and exercise, monotherapy with canagliflozin 100 or $300 \mathrm{mg} / \mathrm{day}$ improved glycaemic control over 26 weeks, with each dosage significantly reducing $\mathrm{HbA}_{1 \mathrm{C}}$ and FPG levels relative to placebo; the proportion of patients achieving an $\mathrm{HbA}_{1 \mathrm{C}}$ target of $<7 \%$ also significantly favoured the canagliflozin groups (Table 1) [13]. Improvements in glycaemic control were sustained over 52 weeks in canagliflozin recipients who continued to receive the drug in the 26-week extension of this trial (Table 1) [33]. Moreover, another study in this setting found canagliflozin 100 or $300 \mathrm{mg} / \mathrm{day}$ to be noninferior to metformin extended-release in improving $\mathrm{HbA}_{1 \mathrm{C}}$ over 26 weeks (Table 1) [27].

Canagliflozin was also an effective add-on therapy in patients with T2D inadequately controlled by their current AHA regimen. Indeed, as an add-on to metformin, canagliflozin 100 or $300 \mathrm{mg} /$ day significantly improved $\mathrm{HbA}_{1 \mathrm{C}}$ and other glycaemic parameters over 26 weeks relative to placebo (Table 2) [34]. Moreover, compared with adding sitagliptin [34] or glimepiride [35] in this setting, adding canagliflozin $100 \mathrm{mg} / \mathrm{day}$ was noninferior and adding canagliflozin $300 \mathrm{mg} /$ day was superior in lowering $\mathrm{HbA}_{1 \mathrm{C}}$ levels over 52 weeks (Table 2). A target $\mathrm{HbA}_{1 \mathrm{C}}$ of $<7.0 \%$ was reached by $41-60 \%$ of patients at 52 weeks in these trials (Table 2) [34, 35], with the odds of achieving this target without concomitant hypoglycaemia being 2.1- and 2.9-fold greater with canagliflozin $100 \mathrm{or} 300 \mathrm{mg} /$ day than with glimepiride (post hoc analysis) [28]. Reductions in FPG were also significantly [34] or numerically [35] greater in the canagliflozin than in the active comparator groups at 52 weeks in these studies (Table 2). Longer term, the relative glycaemic benefits of the canagliflozin and glimepiride regimens were generally sustained over up to 104 weeks' therapy (Table 2) [36].

Similarly, in patients with T2D inadequately controlled by metformin plus either a sulfonylurea [14], pioglitazone [16] or sitagliptin [37], adding canagliflozin (100 or $300 \mathrm{mg} /$ day) significantly lowered both $\mathrm{HbA}_{1 \mathrm{C}}$ and FPG levels and significantly increased the proportion of patients achieving $\mathrm{HbA}_{1 \mathrm{C}}$ levels $<7 \%$ versus placebo over 26 weeks, with these benefits sustained up to 52 weeks $[\underline{14}, \underline{16}]$ (Table 2). Another trial in a similar patient population found adding canagliflozin $300 \mathrm{mg} /$ day to metformin plus a sulfonylurea to be more effective in lowering $\mathrm{HbA}_{1 \mathrm{C}}$ and FPG levels over 52 weeks than adding sitagliptin, and numerically more canagliflozin than sitagliptin recipients achieved $\mathrm{HbA}_{1 \mathrm{C}}<7 \%$ (Table 2) [15].

\subsection{Bodyweight}

Canagliflozin ( 100 or $300 \mathrm{mg} /$ day) significantly reduced bodyweight relative to placebo over 26 weeks, both when used as monotherapy in patients with T2D inadequately controlled by diet and exercise (Table 1) [13] and when used as addon therapy in patients whose T2D was inadequately controlled by metformin, either alone [34] or in combination with another oral AHA [14, 16, 37] (Table 2). In all settings, weight loss was sustained with canagliflozin up to 52 weeks (Table 1 and Table 2) [14, $\underline{16}, \underline{33}, \underline{34]}$.

In active comparator-controlled trials, adding canagliflozin (100 or $300 \mathrm{mg} /$ day) to ongoing metformin monotherapy [34, 35] or metformin plus a sulfonylurea [15] significantly reduced bodyweight over 52 weeks compared with adding either sitagliptin [15, 34] or glimepiride [35], with the benefit of canagliflozin versus glimepiride being maintained over 104 weeks' treatment [36] (Table 2). Notably, in a post hoc analysis [38] of one of these studies [35, 36], more overweight/obese patients (BMI $\geq 25 \mathrm{~kg} / \mathrm{m}^{2}$ ) lost $\geq 4.5 \mathrm{~kg}$ in bodyweight with canagliflozin than with glimepiride over 52 and 104 weeks.

The weight loss associated with canagliflozin seems mainly due to fat mass reduction [35] and, according to a pooled analysis of four phase 3 placebo-controlled studies $(n=2250)$ [39], may contribute to some of the $\mathrm{HbA}_{1 \mathrm{C}^{-}}$(Sect. $\underline{4.1}$ ) and SBP- (Sect. $\underline{4.3}$ ) lowering benefits of the drug $(\approx 15$ and $\approx 42 \%$, respectively). 


\subsection{Other Parameters}

In general, BP was modestly lowered with canagliflozin (as monotherapy or add-on therapy) in the trials discussed so far. For instance, in placebo-controlled studies $[13,14, \underline{16}, \underline{34}, \underline{37]}$, mean changes from baseline $(126-131 \mathrm{mmHg})$ in SBP over 26 weeks ranged from -5.8 to $-3.3 \mathrm{mmHg}$ with canagliflozin ( 100 or $300 \mathrm{mg} / \mathrm{day}$ ) versus -2.7 to $+1.5 \mathrm{mmHg}$ with placebo, with the between-group difference being statistically significant $(p<0.025)$ in all but one trial [14]. Mean changes from baseline $(76-79 \mathrm{mmHg}$ ) in DBP in the respective groups ranged from -3.5 to $-1.7 \mathrm{mmHg}$ and from -1.7 to $+0.3 \mathrm{mmHg}$ ( $p=0.002$ for canagliflozin vs. placebo, where reported/assessed [37]). Moreover, when four 26-week phase 3 placebo-controlled trials were pooled $(n=2313)$, the SBP- and DBP-lowering benefits of canagliflozin were seen regardless of whether antihypertensives were, or were not, taken concomitantly [40], and improvements in mean arterial pressure, pulse pressure and double product (a cardiac workload measure) were also seen with the drug [41].

In active comparator-controlled trials, adding canagliflozin (100 or $300 \mathrm{mg} /$ day) to ongoing metformin monotherapy $[\underline{34}, \underline{35}]$ or metformin plus a sulfonylurea [15] reduced SBP over 52 weeks compared with adding either sitagliptin $(p<$ $0.001)[15, \underline{34}]$ or glimepiride (no p-value; comparison not prespecified) [35], with DBP-lowering benefits also evident in the canagliflozin versus the comparator groups. The SBP- and DBP-lowering effects of canagliflozin were durable, being sustained over 104 weeks of treatment [36].

In terms of lipids, canagliflozin (as monotherapy or add-on therapy) was generally associated with modest increases in HDL-C and LDL-C levels (with minimal LDL-C:HDL-C ratio changes) and modest reductions in triglyceride versus placebo over 26 weeks in key clinical trials (between-group differences were significant/numerical) [푸 $, \underline{14}, \underline{34}, \underline{37}]$; similar findings were generally seen at 52 weeks $[\underline{14}, \underline{16}, \underline{33}]$. In active comparator-controlled trials, adding canagliflozin to metformin-based therapy for 52 weeks appeared to increase HDL-C levels $[\underline{15}, \underline{34}, \underline{35}]$ and (in some studies [15, 35]) LDL-C levels, relative to adding sitagliptin [15, 34] or glimepiride [35]. During this period, canagliflozin also reduced triglyceride levels relative to glimepiride [35], but did not significantly differ from sitagliptin in terms of triglyceride changes $[15, \underline{34}]$. Longer-term data from the glimepiride-controlled trial at 104 weeks were generally consistent with these findings []].

In a post hoc analysis [42] of this trial [36], canagliflozin also appeared to preserve renal function relative to glimepiride over 104 weeks. The annual slope of eGFR decline was significantly $(p<0.01)$ smaller with canagliflozin 100 or $300 \mathrm{mg} /$ day than with glimepiride $\left(0.5\right.$ and $0.9 \mathrm{vs.} 3.3 \mathrm{~mL} / \mathrm{min} / 1.73 \mathrm{~m}^{2}$ ), with canagliflozin $300 \mathrm{mg} /$ day (but not $100 \mathrm{mg} /$ day) also significantly ( $p<0.01$ vs. glimepiride) reducing the urinary albumin : creatinine ratio, independent of $\mathrm{HbA}_{1 \mathrm{C}}$ changes [42].

\subsection{Special Patient Populations}

The findings of trials and subgroup analyses assessing canagliflozin efficacy in various special patient groups are generally consistent with those of the pivotal trials already discussed; efficacy measures of particular relevance are summarized here.

\subsubsection{High CVD Risk Patients}

Clinical efficacy data for canagliflozin in patients with inadequately controlled T2D and an elevated risk of cardiovascular disease (CVD) are available from prespecified [43, 44] and post hoc [45] subgroup analyses of CANVAS, a trial designed primarily to assess the impact of canagliflozin on CVD risk [46]. In these patients, adding canagliflozin 100 or $300 \mathrm{mg}$ /day to insulin $\geq 20 \mathrm{IU} /$ day (with or without other AHAs) significantly improved $\mathrm{HbA}_{1 \mathrm{C}}$ and FPG measures over 18 weeks relative to adding placebo, and these benefits were sustained to week 52 (Table 3); findings were independent of insulin dosage [43]. Similarly, adding canagliflozin 100 or $300 \mathrm{mg} /$ day to ongoing 
sulfonylurea monotherapy [44] or a DPP4 inhibitor or GLP-1 receptor agonist (RA), with or without other AHAs [45], improved glycaemic measures over 18 weeks versus adding placebo, although some subgroups were small (Table 3 ). Over 18-52 weeks, canagliflozin, compared with placebo, reduced bodyweight by up to $3.5 \mathrm{~kg}$ across subgroups (Table 3) [43-45] and, in the largest substudy [43], reduced SBP and DBP and had minimal impact on the LDL-C: HDL-C ratio.

These findings are generally supported by a post hoc pooled analysis of four 26-week placebo-controlled phase 3 trials $(n=2313)$ in which canagliflozin (100 or $300 \mathrm{mg} /$ day) regimens improved glycaemic control, bodyweight and SBP relative to placebo regardless of patient CVD history/risk factors [47].

\subsubsection{Older Patients}

In older patients $(n=714)$ aged 55-80 years with T2D inadequately controlled by oral/injectable AHA regimens, adding canagliflozin 100 or $300 \mathrm{mg} /$ day significantly $(p<0.001)$ improved glycaemic control over 26 weeks versus adding placebo, as measured by mean changes from baseline in $\mathrm{HbA}_{1 \mathrm{C}}(-0.60$ and -0.73 vs. $-0.03 \%$; overall baseline value $7.7 \%$ across groups) and FPG ( -18.1 and $-20.3 \mathrm{vs.}+7.4 \mathrm{mmol} / \mathrm{L}$; overall baseline value $157 \mathrm{mmol} / \mathrm{L})$ levels [48]. Significant $(p<0.001)$ bodyweight loss also occurred with canagliflozin versus placebo during this period (mean changes from baseline -2.2 and -2.8 vs. $-0.1 \mathrm{~kg}$; overall baseline value $90 \mathrm{~kg}$ ) [48]. Longer term, canagliflozin 100 or $300 \mathrm{mg} /$ day largely maintained improvements versus placebo in $\mathrm{HbA}_{1 \mathrm{C}}$ levels (mean changes from baseline: -0.32 and -0.43 vs. $+0.17 \%)$, as well as FPG levels and bodyweight, over 104 weeks in a 78-week extension $(n=624)$ [49].

Notably, canagliflozin (as monotherapy or combination therapy) improved glycaemic control versus placebo regardless of whether patients were aged $\geq 65$ or $<65$ years $(n=445$ and 1868) [50] or $\geq 75$ or $<75$ years $(n=183$ and 3975) [51] in pooled analyses of four [50] or six [51] phase 3 trials (plus CANVAS substudies [51]) of 18-26 weeks' duration. However, glycaemic improvements were slightly more pronounced in the younger patient groups [ $\underline{50}, \underline{51}]$.

\subsubsection{Other Patients}

In Asian patients with T2D, canagliflozin 100 or $300 \mathrm{mg} /$ day, as first-line monotherapy [ $\underline{26}, \underline{52}]$ or added to oral AHA $\left[\underline{26}, \underline{53}\right.$ ] or insulin [ $\underline{54}$ ] regimens, significantly $(p<0.05)$ improved $\mathrm{HbA}_{1 \mathrm{C}}, \mathrm{FPG}$ and bodyweight versus corresponding placebo regimens in phase 3 (or unspecified phase [54]) trials of 16-24 weeks' duration (total $n=146-676$ ), with improvements in these parameters also seen in a 52-week noncomparative study (total $n=1299$; primary endpoint not specified) [26]. Indeed, in post hoc analyses, race did not impact the glycaemic or bodyweight benefits of canagliflozin regimens in placebo-controlled trials (pooled; $n=124-3108$ per racial group) [55-59] or in one $(n=307)$ [56] or three ( $n=551$; pooled) [59] active comparator-controlled studies.

Reductions in $\mathrm{HbA}_{1 \mathrm{C}}$ and bodyweight (of $\approx 0.5$ and $\approx 2 \%$ ) were also seen with canagliflozin (100 or $300 \mathrm{mg} /$ day) versus placebo in T2D patients with renal impairment (eGFR 45 to $\left.<60 \mathrm{~mL} / \mathrm{min} / 1.73 \mathrm{~m}^{2}\right)$ in a pooled analysis $(n=721)$ of phase 3 trials; most (94\%) patients in this analysis were receiving an AHA regimen at baseline [60]. Moreover, in patients with T2D who met metabolic syndrome criteria in two phase 3 trials $(n=1169$ or 586; post hoc analysis), adding canagliflozin 100 or $300 \mathrm{mg} /$ day to ongoing metformin-based therapy generally improved glycaemic $\left(\mathrm{HbA}_{1 \mathrm{C}}\right.$, FPG) as well as non-glycaemic (e.g. bodyweight, BMI, waist circumference, BP, HDL-C) metabolic parameters over 52 weeks relative to adding glimepiride or sitagliptin, although generally increased LDL-C levels [61].

\subsection{Additional Analyses}

Canagliflozin (100 or $300 \mathrm{mg} / \mathrm{day}$ ), as monotherapy or add-on therapy, was effective in lowering $\mathrm{HbA}_{1 \mathrm{C}}$ levels in T2D patients, regardless of baseline patient/disease characteristics such as age [62], BMI [62], $\mathrm{HbA}_{1 \mathrm{C}}$ level [63], T2D 
duration [63] or renal function (although efficacy declined with increasing renal impairment) [62], in post hoc pooled analyses of four $(n=2313)$ [63] or six $(n=4053)$ [62] placebo-controlled phase 3 trials of 18-26 weeks' duration.

Moreover, post hoc analyses of composite endpoints have confirmed the concomitant benefit of canagliflozin on glycaemic and other metabolic parameters (e.g. bodyweight, BP and lipids) in T2D patients [29 , 64-66]. For instance, a reduction in both $\mathrm{HbA}_{1 \mathrm{C}}$ and bodyweight was achieved by 1.5- to 3.6-fold more recipients of canagliflozin (100 or 300 $\mathrm{mg} /$ day) than of placebo [29], glimepiride [66] or sitagliptin [67], when each was used for 18 [29] or 52 [66, 67] weeks as add-on therapy to ongoing AHA regimens in CANVAS [29], CANTATA-SU [66] or two other phase 3 trials (pooled; $n=1856$ ) [67]. Notably, combined reductions in bodyweight and $\mathrm{HbA}_{1 \mathrm{C}}$ with canagliflozin may lead to improvements in liver enzyme levels in patients with T2D, according to additional pooled phase 3 study data [68].

\subsection{Real-World Studies}

The efficacy of canagliflozin in the real-world setting has been established in various analyses of US healthcare claims databases and/or healthcare datasets [30-32, 69-71]. Among those fully published ( $n=826-1562$ evaluable) [69-71], canagliflozin regimens reduced $(p<0.001$, where specified $[69, \underline{71}]) \mathrm{HbA}_{1 \mathrm{C}}$ levels from baseline by a mean of 0.70 $0.81 \%$ and increased the proportion of patients achieving $\mathrm{HbA}_{1 \mathrm{C}}$ levels $<7.0 \%$ by approximately twofold $(p<0.001$ where specified [71]) over 6 months [69] or mean of 67-185 days [70, 71]. Limited glycaemic data available from the other analyses $(n=1227-16,163)$ [30-32] were generally consistent with these findings, with one indicating significantly $(p=0.004)$ greater reductions in $\mathrm{HbA}_{1 \mathrm{C}}$ levels with canagliflozin than with DPP4 inhibitor regimens over $\approx 183$ days' mean follow-up [32]. In another of these analyses (designed primarily to assess bodyweight), canagliflozin regimens were associated with significant $(p<0.0001)$ mean reductions from baseline in bodyweight, ranging from $-1.8 \mathrm{~kg}$ at 3 months to $-2.6 \mathrm{~kg}$ at 12 months in the overall population and -2.1 to $-3.0 \mathrm{~kg}$ in patients with a baseline BMI of $\geq 30 \mathrm{~kg} / \mathrm{m}^{2}[\underline{31}]$.

\section{Tolerability}

Oral canagliflozin, as monotherapy or add-on therapy, was generally well tolerated for up to 104 weeks in patients with T2D, including those of older age and/or at high CV risk, in the key phase 3 or 4 trials discussed in Sect. $\underline{4}$. In the pooled analysis of four placebo-controlled phase 3 trials $(n=2313)$ [72], treatment-related adverse events (AEs) occurred in up to 1.7 -fold more canagliflozin 100 or $300 \mathrm{mg} /$ day than placebo recipients over 26 weeks (20.5 and 22.9 vs. $13.2 \%$ ) and, consistent with canagliflozin's mechanism of action (Sect. 2), the most common AEs associated with the drug were female genital mycotic infection (GMI) (10.4 and 11.4 vs. 3.2\%), urinary tract infection (UTI) (5.9 and 4.3 vs. $4.0 \%$ ), increased urination ( 5.3 and 4.6 vs. $0.8 \%$ ) and male GMI (4.2 and 3.7 vs. $0.6 \%$ ). AEs were generally mild or moderate and few patients experienced serious AEs $(\approx 3 \%$ in each group) or death $(<0.3 \%$ in each group) [72].

In active comparator-controlled studies, canagliflozin regimens were generally similar to sitagliptin or glimepiride regimens in terms of the incidence of treatment-related AEs (20-34 vs. 20-28\%) and discontinuations because of AEs (3-7 vs. 3-6\%) over 52 weeks $[\underline{15}, \underline{34}, \underline{35}]$, with 104-week data from the glimepiride-controlled trial being consistent with these findings [36]. Local prescribing information should be consulted for warnings and precautions relating to AEs such as diabetic ketoacidosis (rare but sometimes serious/fatal [ $7, \underline{73}]$ ), elevated haematocrit, and GMIs [7].

\subsection{Genitourinary Infections}

Canagliflozin increases UGE (Sect. 2), which may contribute to GMIs [7]. Canagliflozin $300 \mathrm{mg} /$ day significantly ( $p<$ $0.00001 ; 3.76$-fold) increased the risk of GMIs versus placebo over 12-26 weeks in a meta-analysis of eight placebocontrolled trials $(n=1338)$ [74]. The most common GMIs with canagliflozin (100 or $300 \mathrm{mg} / \mathrm{day})$ over 26 weeks 
included vulvovaginal mycotic infection in women (5.9 and 5.3 vs. $1.3 \%$ with placebo) and balantitis in men (2.2 and 1.7 vs. $0 \%$ ) in the pooled analysis of four placebo-controlled studies [72]. GMIs in this analysis were never serious, rarely $(<1 \%$ of patients) led to therapy discontinuation and responded to standard antifungal treatment [72], lasting a median of 7 and 18 days in treated female and male canagliflozin recipients [75].

The likelihood of GMIs with canagliflozin $300 \mathrm{mg} / \mathrm{day}$ was significantly greater (4.95-fold; $p<0.00001)$ than with sitagliptin or glimepiride over $12-52$ weeks in a meta-analysis of four active comparator-controlled trials $(n=2510)$ [74]. Similarly, in individual studies, the GMI incidence was numerically greater with canagliflozin (100 or 300 $\mathrm{mg} / \mathrm{day})$ than with sitagliptin or glimepiride regimens over 52 weeks both in men (2-9 vs. $0.5-1 \%)$ and women (10-15 vs. 2-4\%) [15, 34, 35], although did not further increase versus glimepiride over 104 weeks [36].

Despite being a common AE with canagliflozin, UTIs did not significantly differ in incidence between canagliflozin $300 \mathrm{mg} /$ day and placebo over 12-26 weeks in the meta-analysis of eight trials [74]. UTIs with canagliflozin (100 or 300 $\mathrm{mg} /$ day), although often symptomatic, were rarely serious $(\leq 0.2 \%$ of patients) [76] and responded to standard therapy without canagliflozin discontinuation [7] when four placebo-controlled studies were pooled. Compared with other active agents, the UTI incidence with canagliflozin did not significantly differ from that with sitagliptin or glimepiride in the meta-analysis of four 12- to 52-week trials [74], with data from individual sitagliptin- or glimepiride-controlled studies of up to 104 weeks' duration generally supporting these findings [15, 34-36].

\subsection{Osmotic Diuresis and Volume Depletion}

By increasing UGE, canagliflozin can trigger osmotic diuresis. Treatment-related AEs related to osmotic diuresis (e.g. increased urine volume/frequency) occurred in 6.1-fold more canagliflozin (100 or $300 \mathrm{mg} / \mathrm{day})$ than placebo recipients ( 4.9 and 4.9 vs. $0.8 \%)$ in the pooled analysis of four 26-week trials $(n=2313)$ [40]; these AEs typically occurred during the first 6 weeks of therapy and none were serious [72]. Consistent with these findings, over 12-52 weeks, canagliflozin significantly $(p<0.01)$ increased the risk of osmotic diuresis-related AEs compared with placebo, as well as active comparators (sitagliptin or glimepiride), in meta-analyses ( $n=3853$ and 5057) [74].

AEs related to volume depletion (e.g. postural dizziness, orthostatic hypotension) were rare $(\approx 1 \%$ incidence) with canagliflozin in the pooled analysis of four placebo-controlled trials [40] and occurred predominantly in patients on antihypertensives [72]. Moreover, these AEs did not significantly differ in incidence between canagliflozin and placebo or active comparators (sitagliptin or glimepiride) in meta-analyses $(n=3334$ and 4910) [74]. When risk factors for volume depletion-related AEs were assessed in a pooled analysis of eight phase 3 trials $(n=9439)$, the incidence was generally numerically greater with canagliflozin 100 or $300 \mathrm{mg}$ /day than with comparators in patients who were receiving loop diuretics (3.2 and 8.8 vs. $4.7 \%$ ), had a baseline eGFR of 30 to $<60 \mathrm{~mL} / \mathrm{min} / 1.73 \mathrm{~m}^{2}(4.8$ and 8.1 vs. $2.6 \%$ ) or were aged $\geq 75$ years ( 4.9 and 8.7 vs. $2.6 \%$ ) [7]. Similarly, in CANVAS (in which patients generally had more T2D complications), the incidence of volume depletion-related AEs was 2.8 and $4.6 \%$ with canagliflozin 100 or 300 $\mathrm{mg}$ /day versus $1.9 \%$ with placebo [7]. However, canagliflozin did not increase the incidence of volume depletionrelated AEs that were serious or that led to discontinuation in these studies [7].

Volume depletion with canagliflozin may reduce eGFR, although the reductions are usually small, occur in the first few weeks of therapy $[\underline{7}, \underline{72}]$ and stabilize/attenuate thereafter $[\underline{72}, \underline{77}]$. However, large $(>30 \%)$, albeit transient, eGFR reductions have occurred with canagliflozin in patients more susceptible to volume depletion (such as the high-risk patients already discussed), although did not usually require treatment interruption [7]. Renal-related AEs (e.g. reduced GFR, increased blood creatinine) occurred with an incidence of $<3 \%$ and were rarely serious $(\leq 0.2 \%$ of patients) with canagliflozin or comparators over 26 [72] or 104 [78] weeks' therapy in pooled analyses of placebo- and/or active comparator-controlled trials $[\underline{72}, \underline{78}]$. Nevertheless, a possible signal for acute renal injury was detected with 
canagliflozin, as well as other SGLT2 inhibitors, when postmarketing data from the US FDA AE Reporting System were assessed [78].

\subsection{Hypoglycaemia}

Hypoglycaemia was relatively uncommon when canagliflozin (100 or $300 \mathrm{mg} /$ day) was used as monotherapy [13,$\underline{33}]$ or added to metformin (alone [34] or in combination with sitagliptin [37] or pioglitazone [16]) over 26 weeks (3-4 vs. $2-3 \%$ with placebo) or 52 weeks (4-7\%) in placebo- and/or active comparator-controlled trials [13, $\underline{34}, \underline{37}]$ and their extensions [16, 33]. In patients receiving metformin in active comparator-controlled studies, the incidence of hypoglycaemia with add-on canagliflozin (100 or $300 \mathrm{mg} /$ day) was not markedly different from that with add-on sitagliptin (7 and 7 vs. 4\%) [34] but was significantly $(p<0.0001)$ lower than with add-on glimepiride (6 and 5 vs. $34 \%$ ) [35] over 52 weeks, with the benefit over glimepiride maintained at week 104 [36]. Severe hypoglycaemia was rare $(<1 \%)$ with canagliflozin in these trials, where specified [13, $16, \underline{34-37]}$.

By contrast, hypoglycaemia tended to be relatively common when canagliflozin was added to an AHA regimen that included a sulfonylurea [14, 15, 44] or insulin [43] in phase 3 trials. For instance, in patients receiving metformin plus a sulfonylurea, the incidence of hypoglycaemia over 52 weeks was approximately twofold greater with add-on canagliflozin 100 or $300 \mathrm{mg}$ /day than with add-on placebo (34 and 37 vs. 18\%) [14] but did not markedly differ between add-on canagliflozin $300 \mathrm{mg} /$ day and sitagliptin (43 vs. 41\%) [15]; severe hypoglycaemia was not common ( $\leq 4 \%$ incidence) in any treatment group of either trial. Added to insulin therapy, canagliflozin $100 \mathrm{or} 300 \mathrm{mg} / \mathrm{day}$ did not significantly differ from placebo in terms of hypoglycaemia (59 and 57 vs. $48 \%$ ) or severe hypoglycaemia (5 and 6 vs. $4 \%$ ) incidence over 52 weeks in CANVAS [녀]

\subsection{Cardiovascular and Other Events}

Canagliflozin (100 or $300 \mathrm{mg} /$ day, pooled) did not increase the risk of major CV events (MACE) relative to placebo and active comparators (combined) in a pre-specified interim meta-analysis of phase 2 or 3 trials ( $n=9632 ; 44.9 \%$ were high CV risk patients from CANVAS). The hazard ratio (HR) was $0.91(95 \% \mathrm{CI} 0.68,1.22)$ for the composite primary endpoint of time to $\mathrm{CV}$ death, non-fatal stroke, non-fatal myocardial infarction and unstable angina requiring hospitalization [7].

Lower limb amputations (mainly of the toes) appeared to increase in incidence with canagliflozin in T2D patients with, or at high risk of, CVD in ongoing long-term trials [7]. For instance, in an interim safety analysis of CANVAS (mean follow-up 4.5 years), lower limb amputation occurred with an approximately twofold greater incidence with canagliflozin 100 or $300 \mathrm{mg} /$ day than with placebo ( 7 and 5 vs. 3 per 1000 patient-years) [79]. The mechanism underlying this risk has not yet been determined; patients at higher risk of amputation should be monitored and counselled appropriately, and canagliflozin may need to be discontinued if events such as skin ulcer, infection, osteomyelitis or gangrene develop in the lower extremities [7].

In addition, bone fracture risk was significantly increased with canagliflozin (100 or $300 \mathrm{mg} / \mathrm{day}$, combined) relative to placebo over 104 weeks in CANVAS ( $n=4327$ ) (4.0 vs. $2.6 \%$ of patients; HR $1.51 ; 95 \%$ CI 1.04, 2.19), whereas pooled data from non-CANVAS studies found no significant fracture risk with these canagliflozin dosages over $52(n=$ 5867) or $104(n=2164)$ weeks versus placebo/active agents (combined) [80]. The reason for the increased fracture risk with canagliflozin in CANVAS but not the non-CANVAS studies is unknown, although differences in factors such as patient age (mean 62 vs. 58 years), loop diuretic use (12 vs. $4 \%$ of patients) and eGFR (mean $77 \mathrm{vs.} 85 \mathrm{~mL} / \mathrm{min}$ ) have been suggested. 


\section{Dosage and Administration}

In the EU, canagliflozin is approved for use as monotherapy (as an adjunct to diet and exercise, when metformin is considered inappropriate) and as an add-on therapy (to other AHAs, including insulin) to improve glycaemic control in adults with T2D [7]. Canagliflozin tablets should be taken orally, preferably prior to the first food of the day. The initial dosage is $100 \mathrm{mg}$ once daily; if tolerated (and eGFR is $\geq 60 \mathrm{~mL} / \mathrm{min} / 1.73 \mathrm{~m}^{2}$ ), this can be increased to $300 \mathrm{mg}$ once daily if necessary. Care is advised if increasing the dosage in patients for whom the initial diuresis associated with the drug may pose a risk (e.g. those aged $\geq 75$ years or with known CVD) [7]. Local prescribing information should be consulted for further details, including drug interactions, use in special patient populations, contraindications and other warnings and precautions.

\section{Place of Canagliflozin in T2D Management}

Managing T2D requires an individualized stepwise approach [1, 2], taking into consideration common patient comorbidities (e.g. heart failure, coronary artery disease) and the likelihood that AHA-associated hypoglycaemia (thought to contribute to CV dysfunction and, in high-risk patients, CV events) may have untoward outcomes [2] . Among the numerous AHAs now available, metformin monotherapy remains the standard first-line option for most patients $[1, \underline{2}]$, although sequential addition of drugs from other classes is often required to attain/maintain good glycaemic control.

Although most AHAs lower blood glucose levels by increasing insulin secretion and/or sensitivity, SGLT2 inhibitors (e.g. canagliflozin, dapagliflozin and empagliflozin [81]) act independently of insulin, enabling them to be used at any stage of T2D (i.e. regardless of insulin secretory capacity) and to complement a wide variety of AHAs as part of combination regimens [2, 82]. In treatment guidelines, SGLT2 inhibitors (as well as sulfonylureas, thiazolidinediones, DPP4 inhibitors, GLP-1 RAs and insulin) are generally recommended as second- and/or subsequentline options for use in combination regimens, although can be used first line if metformin is contraindicated/not tolerated $[\underline{1}, \underline{2}]$ (provided a sulfonylurea or pioglitazone is inappropriate and a DPP4 inhibitor would otherwise be used [83]).

Canagliflozin is one of the most widely available SGLT2 inhibitors [81]. Its approval as a first-line monotherapy or as an add-on to other AHAs, including insulin, in adults with T2D (Sect. $\underline{6}$ ) was based on numerous well-designed clinical trials in these settings, in which the drug (at 100 or $300 \mathrm{mg} /$ day) provided improved and sustainable glycaemic control over up to 104 weeks' therapy (Sect. 4). The glycaemic efficacy of canagliflozin $100 \mathrm{mg} /$ day was noninferior to that of metformin as first-line monotherapy and to that of sitagliptin or glimepiride as an add-on therapy, whereas canagliflozin $300 \mathrm{mg} /$ day was more effective than sitagliptin or glimepiride in the add-on setting (Sect. 4.1). Real-world data are also now available and support the use of canagliflozin in T2D management (Sect. 4.6).

In addition to hyperglycaemia, the common comorbidities of T2D, such as obesity, hypertension and dyslipidaemia, should also be addressed to minimize the overall CV risk of T2D [84]. Canagliflozin (like other SGLT2 inhibitors) induces moderate bodyweight loss (Sect. 4.2) through urinary loss of glucose (and thus calories) (Sect. $\underline{2})[\underline{2}, \underline{82}]$. The ability to reduce bodyweight is shared by few other AHAs (including GLP-1 RAs), with most increasing bodyweight (e.g. sulfonylureas, meglitinides, thiazolidinediones and insulin) or being bodyweight neutral (e.g. DPP4 inhibitors, metformin, $\alpha$-glucosidase inhibitors) $[\underline{2}, \underline{85}]$; as such, canagliflozin has a bodyweight profile more favourable than that of glimepiride or sitagliptin as an add-on therapy (Sect. 4.2). Bodyweight losses occur with canagliflozin even in combination with AHAs typically associated with bodyweight gain, and appear primarily due to reductions in fat (Sect. 4.2), which could (through improved insulin sensitivity) contribute to the glycaemic benefits of the drug. 
Canagliflozin also appears to modulate various other non-glycaemic CVD risk factors, consistent with the SGLT2 inhibitor class [2]. For instance, the drug generally improved BP (Sect. 4.3) (possibly through weight loss, osmotic diuresis and/or mild natriuresis [86]), serum uric acid levels (Sect. 2) (possibly via increased urinary secretion) and markers of arterial stiffness (Sect. 4.3). It also modestly impacted serum lipid levels (generally increasing HDL-C and LDL-C and reducing triglyceride; Sect. 4.3), although it is not yet clear whether the net impact of the drug on lipids is of any clinical relevance.

However, the CV profile of canagliflozin is being investigated. At present, CV data for the drug are limited to a meta-analysis of T2D studies (including the large ongoing CV outcomes trial CANVAS), which demonstrated no increased MACE risk with canagliflozin (Sect. 5.4). Mature CV data from CANVAS are required to determine whether canagliflozin improves major CV outcomes in T2D patients at high CV risk, a benefit that has been seen with empagliflozin in a similar trial; to date, the only other AHAs to have demonstrated a reduction in MACE risk are GLP-1 RAs [87]. Thus, CANVAS CV data are awaited with interest.

Canagliflozin is generally well tolerated and, consistent with its mechanism of action, the most common AEs are genitourinary infections and increased urination (Sect. 5). As with other SGLT2 inhibitors and most other AHAs [2], hypoglycaemia is uncommon with canagliflozin, unless used in combination with drugs that increase the risk of the event (Sect. 5.3), among which are sulfonylureas, insulins and meglitinides [2].

Reductions in eGFR initially occur with canagliflozin due to volume depletion and may explain the signal of acute kidney injury identified with the drug (and other SGLT2 inhibitors) postmarketing (Sect. 5.2). However, over 104 weeks, eGFR reductions with canagliflozin were less than those with glimepiride, suggesting kidney function preservation (Sect. 4.3), possibly via albuminuria and glomerular hyperfiltration attenuation [78]. Notably, the effects of the drug on renal and CV outcomes in T2D patients with diabetic nephropathy (CREDENCE; NCT02065791) or increased risk of CV events (CANVAS-R; NCT01989754) are currently being evaluated, as are its effects in T2D patients with congestive heart failure (CANDLE; UMIN000017669). Other AEs related to volume depletion (such as dizziness and orthostatic hypotension) are generally uncommon with canagliflozin, but may limit its use in patients particularly susceptible to volume depletion, such as the elderly or those on antihypertensives or with CVD (Sect. 5.2); thus, any volume depletion should be corrected before initiating canagliflozin [7]. Further longer-term studies evaluating the benefits versus potential risks of canagliflozin and other SGLT2 inhibitors would be beneficial, including the potential for lower-limb amputations and bone fractures (Sect. 5.4).

Also of interest are robust trials directly comparing canagliflozin with AHAs such as other SGLT2 inhibitors or GLP-1 RAs. Currently, such comparisons are limited to network meta-analyses, across which canagliflozin was at least as effective in improving glycaemic control over 26 (or 26-104 [88]) weeks as empagliflozin [88-90], dapagliflozin [88-90], sitagliptin, pioglitazone or a sulfonylurea [90], when used as monotherapy [90] or as part of a dual [88] or triple [89] AHA regimen. Similar comparisons (including those vs. GLP-1 RAs) had more mixed findings, depending on the canagliflozin/comparator dosage and the timepoint and/or treatment setting assessed [91, 92]. Due to their indirect nature, findings from such analyses should be interpreted with caution.

Like most AHAs, canagliflozin and other SGLT2 inhibitors have the convenience of oral administration (unlike GLP-1 RAs and insulins, which are injectable), although the cost of SGLT inhibitors and other relatively recent AHA classes (e.g. DPP4 inhibitors, GLP-1 RAs) is high [2]. Various canagliflozin cost-utility analyses conducted from the NHS perspective of the UK [푸 94] or Spain [95, 96] are available. Their findings suggest that canagliflozin $100 \mathrm{mg}$ may dominate (i.e. be cheaper with greater quality-adjusted life-year gains) and canagliflozin $300 \mathrm{mg}$ may be cost effective versus both empagliflozin $25 \mathrm{mg}$ and dapagliflozin $10 \mathrm{mg}$ as monotherapy [94] and versus sitagliptin $100 \mathrm{mg}$ as an add-on to metformin [96]. Both canagliflozin dosages may also dominate dapagliflozin $10 \mathrm{mg}$ [95] and be cost effective versus a sulfonylurea [93] in the latter setting, and dominate sitagliptin as an add-on to metformin plus a sulfonylurea [96]. Additional cost analyses conducted in the UK [97] and from an Italian NHS perspective [98] 
generally support these findings, with canagliflozin estimated to be cost saving versus other SGLT inhibitors [97], as well as sitagliptin and glimepiride [98], when used as add-on therapy. Further cost-utility analyses would be beneficial.

In conclusion, canagliflozin is an effective and generally well tolerated once-daily oral AHA for T2D management, with an insulin-independent mechanism of action that makes it a particularly useful option for use in combination regimens. Given the drug improves glycaemic control, as well as bodyweight and BP, and has a low risk of hypoglycaemia, its CV profile is highly anticipated.

\begin{abstract}
Data selection sources: Database(s): EMBASE, MEDLINE and PubMed from 1946 to present. Clinical trial registries/databases and websites were also searched for relevant data [searches last updated 12 May 2017]. Records were limited to those in English language.
\end{abstract}

Search terms: Canagliflozin, Canaglu, Invokana, JNJ-

28431754, TA-7284, type 2, type II, T2DM, T2D

\section{Acknowledgments}

\section{Compliance with Ethical Standards}

Funding The preparation of this review was not supported by any external funding.

Conflicts of interest Emma Deeks is a salaried employee of Adis/Springer, is responsible for the article content and declares no relevant conflicts of interest.

\section{References}

1. NICE. Type 2 diabetes in adults: management. 2015. http://www.nice.org. Accessed 3 May 2017.

2. Inzucchi SE, Bergenstal RM, Buse JB, et al. Management of hyperglycemia in type 2 diabetes, 2015: a patientcentered approach: update to a position statement of the American Diabetes Association and the European Association for the Study of Diabetes. Diabetes Care. 2015 Jan;38(1):140-9.

3. Ohgaki R, Wei L, Yamada K, et al. Interaction of the sodium/glucose cotransporter (SGLT) 2 inhibitor canagliflozin with SGLT1 and SGLT2: inhibition kinetics, sidedness of action, and transporter-associated incorporation accounting for its pharmacodynamic and pharmacokinetic features. J Pharmacol Exp Ther. 2016 July;358(1):94-102.

4. Nomura S, Sakamaki S, Hongu M, et al. Discovery of canagliflozin, a novel C-glucoside with thiophene ring, as sodium-dependent glucose cotransporter 2 inhibitor for the treatment of type 2 diabetes mellitus. J Med Chem. 2010 Sep 9;53(17):6355-60.

5. Devineni D, Curtin CR, Polidori D, et al. Pharmacokinetics and pharmacodynamics of canagliflozin, a sodium glucose co-transporter 2 inhibitor, in subjects with type 2 diabetes mellitus. J Clin Pharmacol. 2013 Jun;53(6):601-10. 6. Sha S, Devineni D, Ghosh A, et al. Pharmacodynamic effects of canagliflozin, a sodium glucose co-transporter 2 inhibitor, from a randomized study in patients with type 2 diabetes. PLoS ONE. 2014;9(9):e110069.

7. Janssen-Cilag International NV. Invokana film-coated tablets: summary of product characteristics. 2016. http://www.ema.europe.eu. Accessed 18 May 2017.

8. Tanaka H, Takano K, Iijima H, et al. Factors affecting canagliflozin-induced transient urine volume increase in patients with type 2 diabetes mellitus. Adv Ther. 2016 Dec 15;34(2):436-51.

9. Oguma T, Nakayama K, Kuriyama C, et al. Intestinal Sodium Glucose Cotransporter 1 Inhibition Enhances Glucagon-Like Peptide-1 Secretion in Normal and Diabetic Rodents. The Journal of pharmacology and experimental therapeutics. 2015 Sep;354(3):279-89. 
10. Stein P, Berg JK, Morrow L, et al. Canagliflozin, a sodium glucose co-transporter 2 inhibitor, reduces post-meal glucose excursion in patients with type 2 diabetes by a non-renal mechanism: results of a randomized trial. Metabolism. 2014 Oct;63(10):1296-303.

11. Polidori D, Sha S, Mudaliar S, et al. Canagliflozin lowers postprandial glucose and insulin by delaying intestinal glucose absorption in addition to increasing urinary glucose excretion: results of a randomized, placebo-controlled study. Diabetes Care. 2013 Aug;36(8):2154-61.

12. Sha S, Polidori D, Farrell K, et al. Pharmacodynamic differences between canagliflozin and dapagliflozin: results of a randomized, double-blind, crossover study. Diabetes Obes Metab. 2015 Feb;17(2):188-97.

13. Stenlöf K, Cefalu WT, Kim KA, et al. Efficacy and safety of canagliflozin monotherapy in subjects with type 2 diabetes mellitus inadequately controlled with diet and exercise. Diabetes Obes Metab. 2013 Apr;15(4):372-82. 14. Wilding JP, Charpentier G, Hollander P, et al. Efficacy and safety of canagliflozin in patients with type 2 diabetes mellitus inadequately controlled with metformin and sulphonylurea: a randomised trial. Int J Clin Pract. 2013 Dec;67(12):1267-82.

15. Schernthaner G, Gross JL, Rosenstock J, et al. Canagliflozin compared with sitagliptin for patients with type 2 diabetes who do not have adequate glycemic control with metformin plus sulfonylurea: a 52-week randomized trial. Diabetes Care. 2013 Sep;36(9):2508-15.

16. Forst T, Guthrie R, Goldenberg R, et al. Efficacy and safety of canagliflozin over 52 weeks in patients with type 2 diabetes on background metformin and pioglitazone. Diabetes Obes Metab. 2014;16(5):467-77.

17. Polidori D, Mari A, Ferrannini E. Canagliflozin, a sodium glucose co-transporter 2 inhibitor, improves model-based indices of beta cell function in patients with type 2 diabetes. Diabetologia. 2014 Mar 1;57(5):891-901.

18. Weir MR, Kline I, Xie J, et al. Effect of canagliflozin on serum electrolytes in patients with type 2 diabetes in relation to estimated glomerular filtration rate (eGFR). Curr Med Res Opin. 2014 Sep;30(9):1759-68.

19. Gilbert RE, Mende C, Vijapurkar U, et al. Effects of canagliflozin on serum magnesium in patients with type 2 diabetes mellitus: a post hoc analysis of randomized controlled trials. Diabetes therapy. 2017 Apr;8(2):451-8.

20. Davies MJ, Trujillo A, Vijapurkar U, et al. Effect of canagliflozin on serum uric acid in patients with type 2 diabetes mellitus. Diabetes Obes Metab. 2015;17(4):426-9.

21. Bilezikian JP, Watts NB, Usiskin K, et al. Evaluation of bone mineral density and bone biomarkers in patients with type 2 diabetes treated with canagliflozin. J Clin Endocrinol Metab. 2016;101(1):44-51.

22. Devineni D, Murphy J, Wang SS, et al. Absolute oral bioavailability and pharmacokinetics of canagliflozin: a microdose study in healthy participants. Clin Pharmacol Drug Dev. 2015 Jul;4(4):295-304.

23. Devineni D, Manitpisitkul P, Murphy J, et al. Effect of food on the pharmacokinetics of canagliflozin, a sodium glucose co-transporter 2 inhibitor, and assessment of dose proportionality in healthy participants. Clin Pharmacol Drug Dev. 2015 Jul;4(4):279-86.

24. Francke S, Mamidi RN, Solanki B, et al. In vitro metabolism of canagliflozin in human liver, kidney, intestine microsomes, and recombinant uridine diphosphate glucuronosyltransferases (UGT) and the effect of genetic variability of UGT enzymes on the pharmacokinetics of canagliflozin in humans. J Clin Pharmacol. 2015 Sep;55(9):1061-72. 25. Devineni D, Curtin CR, Marbury TC, et al. Effect of hepatic or renal impairment on the pharmacokinetics of canagliflozin, a sodium glucose co-transporter 2 inhibitor. Clin Ther. 2015;37(3):610-28.e4.

26. Inagaki N, Kondo K, Yoshinari T, et al. Efficacy and safety of canagliflozin alone or as add-on to other oral antihyperglycemic drugs in Japanese patients with type 2 diabetes: a 52-week open-label study. J Diabetes Investig. 2015;6(2):210-8.

27. Jodar E, Rosenstock J, Chuck L, et al. Efficacy and safety of canagliflozin monotherapy: results from 2 studies in patients with type 2 diabetes mellitus [abstract no. 734]. Diabetologia. 2015;58(Suppl 1):S350-1

28. Vercruysse F, Davies MJ, Merton K, et al. Achievement of glycaemic goals without hypoglycaemia with canagliflozin versus glimepiride in patients with type 2 diabetes [abstract no. 714]. Diabetologia. 2016;59(Suppl 1):S339-40

29. Woo V, Wysham C, Mathieu C, et al. Canagliflozin reduces both HbA1c and body weight in patients with type 2 diabetes on background dipeptidyl peptidase-4 inhibitors or glucagon-like peptide-1 agonists [abstract no. 186]. Diabetologia. 2015;58(Suppl 1):S93-4

30. Lefebvre P, Pilon D, Robitaille M, et al. Glycated hemoglobin (HbA1c) control in patients with type 2 diabetes mellitus (T2DM) treated with canagliflozin in a realworld setting [abstract no. PDB27]. Value Health. 2015;18(3):A57 
31. Lefebvre P, Chow W, Pilon D, et al. Real-world evaluation of weight loss in patients with type 2 diabetes mellitus treated with canagliflozin - an electronic health record-based study [abstract no. PDB7]. Value Health. 2016;19(3):A198 32. Canovatchel W, Thayer S, Chow W, et al. Efficacy of canagliflozin versus dipeptidyl peptidase-4 inhibitors in patients with type 2 diabetes: results from randomised controlled trials and a real-world study [abstract no. 709]. Diabetologia. 2016;59(Suppl 1):S337

33. Stenlöf K, Cefalu WT, Kim KA, et al. Long-term efficacy and safety of canagliflozin monotherapy in patients with type 2 diabetes inadequately controlled with diet and exercise: findings from the 52-week CANTATA-M study. Curr Med Res Opin. 2014 Sep 30;30(2):163-75.

34. Lavalle-Gonzalez FJ, Januszewicz A, Davidson J, et al. Efficacy and safety of canagliflozin compared with placebo and sitagliptin in patients with type 2 diabetes on background metformin monotherapy: a randomised trial.

Diabetologia. 2013 Sep 13;56(12):2582-92.

35. Cefalu WT, Leiter LA, Yoon K-H, et al. Efficacy and safety of canagliflozin versus glimepiride in patients with type 2 diabetes inadequately controlled with metformin (CANTATA-SU): 52 week results from a randomised, doubleblind, phase 3 non-inferiority trial. Lancet. 2013 Sep 14;382(9896):941-50.

36. Leiter LA, Yoon KH, Arias P, et al. Canagliflozin provides durable glycemic improvements and body weight reduction over 104 weeks versus glimepiride in patients with type 2 diabetes on metformin: a randomized, double-blind, phase 3 study. Diabetes Care. 2015;38(3):355-64.

37. Rodbard HW, Seufert J, Aggarwal N, et al. Efficacy and safety of titrated canagliflozin in patients with type 2 diabetes mellitus inadequately controlled on metformin and sitagliptin. Diabetes Obes Metab. 2016 Aug;18(8):812-9. 38. Patel CA, Bailey RA, Vijapurkar U, et al. A post-hoc analysis of the comparative efficacy of canagliflozin and glimepiride in the attainment of type 2 diabetes-related quality measures. BMC Health Serv Res. 2016 Aug $05 ; 16(\mathrm{a}): 356$.

39. Cefalu WT, Stenlof K, Leiter LA, et al. Effects of canagliflozin on body weight and relationship to HbA1c and blood pressure changes in patients with type 2 diabetes. Diabetologia. 2015 Jun;58(6):1183-7.

40. Weir MR, Januszewicz A, Gilbert RE, et al. Effect of canagliflozin on blood pressure and adverse events related to osmotic diuresis and reduced intravascular volume in patients with type 2 diabetes mellitus. J Clin Hypertens. 2014 Dec;16(12):875-82.

41. Pfeifer M, Townsend RR, Davies MJ, et al. Effects of canagliflozin, a sodium glucose co-transporter 2 inhibitor, on blood pressure and markers of arterial stiffness in patients with type 2 diabetes mellitus: a post hoc analysis. Cardiovasc Diabetol. 2017 Feb 27;16(1):29.

42. Heerspink HJ, Desai M, Jardine M, et al. Canagliflozin slows progression of renal function decline independently of glycemic effects. J Am Soc Nephrol. 2017 Jan;28(1):368-75.

43. Neal B, Perkovic V, de Zeeuw D, et al. Efficacy and safety of canagliflozin, an inhibitor of sodium-glucose cotransporter 2, when used in conjunction with insulin therapy in patients with type 2 diabetes. Diabetes Care. 2015;38(3):403-11.

44. Fulcher G, Matthews DR, Perkovic V, et al. Efficacy and safety of canagliflozin used in conjunction with sulfonylurea in patients with type 2 diabetes mellitus: a randomized, controlled trial. Diabetes Ther. 2015 Sep;6(3):289302 .

45. Fulcher G, Matthews DR, Perkovic V, et al. Efficacy and safety of canagliflozin when used in conjunction with incretin-mimetic therapy in patients with type 2 diabetes. Diabetes Obes Metab. 2016;18(1):82-91.

46. Neal B, Perkovic V, de Zeeuw D, et al. Rationale, design, and baseline characteristics of the canagliflozin cardiovascular assessment study (CANVAS) - a randomized placebo-controlled trial. Am Heart J. 2013 Aug;166(2):217-23.e11.

47. Davies MJ, Merton K, Vijapurkar U, et al. Efficacy and safety of canagliflozin in patients with type 2 diabetes based on history of cardiovascular disease or cardiovascular risk factors: a post hoc analysis of pooled data. Cardiovasc Diabetol. 2017 Mar 21;16(1):40.

48. Bode B, Stenlöf K, Sullivan D, et al. Efficacy and safety of canagliflozin treatment in older subjects with type 2 diabetes mellitus: a randomized trial. Hosp Pract (1995). 2013 Apr;41(2):72-84.

49. Bode B, Stenlof K, Harris S, et al. Long-term efficacy and safety of canagliflozin over 104 weeks in patients aged 55-80 years with type 2 diabetes. Diabetes Obes Metab. 2015;17(3):294-303. 
50. Sinclair A, Bode B, Harris S, et al. Efficacy and safety of canagliflozin compared with placebo in older patients with type 2 diabetes mellitus: a pooled analysis of clinical studies BMC Endocr Disord. 2014;14(1):37.

51. Sinclair AJ, Bode B, Harris S, et al. Efficacy and safety of canagliflozin in individuals aged 75 and older with type 2 diabetes mellitus: a pooled analysis. J Am Geriatr Soc. 2016 Mar;64(3):543-52.

52. Inagaki N, Kondo K, Yoshinari T, et al. Efficacy and safety of canagliflozin monotherapy in Japanese patients with type 2 diabetes inadequately controlled with diet and exercise: a 24-week, randomized, double-blind, placebocontrolled, phase III study. Expert Opin Pharmacother. 2014 Aug;15(11):1501-15.

53. Ji L, Han P, Liu Y, et al. Canagliflozin in Asian patients with type 2 diabetes on metformin alone or metformin in combination with sulphonylurea. Diabetes Obes Metab. 2015;17(1):23-31.

54. Inagaki N, Harashima S, Maruyama N, et al. Efficacy and safety of canagliflozin in combination with insulin: a double-blind, randomized, placebo-controlled study in Japanese patients with type 2 diabetes mellitus. Cardiovasc Diabetol. 2016;15:89.

55. Gavin JR, Davies MJ, Davies M, et al. The efficacy and safety of canagliflozin across racial groups in patients with type 2 diabetes mellitus. Curr Med Res Opin. 2015;31(9):1693-702.

56. John M, Cerdas S, Violante R, et al. Efficacy and safety of canagliflozin in patients with type 2 diabetes mellitus living in hot climates. Int J Clin Pract. 2016;70(9):775-85.

57. Prasanna Kumar KM, Mohan V, Sethi B, et al. Efficacy and safety of canagliflozin in patients with type 2 diabetes mellitus from India. Indian J Endocrinol Metab. 2016 May-Jun;20(3):372-80.

58. Davidson JA, Aguilar R, Lavalle Gonzalez FJ, et al. Efficacy and safety of canagliflozin in type 2 diabetes patients of different ethnicity. Ethn Dis. 2016 Apr 21;26(2):221-8.

59. Lavalle-Gonzalez FJ, Eliaschewitz FG, Cerdas S, et al. Efficacy and safety of canagliflozin in patients with type 2 diabetes mellitus from Latin America. Curr Med Res Opin. 2016;32(3):427-39.

60. Yamout H, Perkovic V, Davies M, et al. Efficacy and safety of canagliflozin in patients with type 2 diabetes and stage 3 nephropathy. Am J Nephrol. 2014;40(1):64-74.

61. Davies MJ, Merton KW, Vijapurkar U, et al. Canagliflozin improves risk factors of metabolic syndrome in patients with type 2 diabetes mellitus and metabolic syndrome. Diabetes Metab Syndr Obes. 2017;10:47-55.

62. Gilbert RE, Weir MR, Fioretto P, et al. Impact of age and estimated glomerular filtration rate on the glycemic efficacy and safety of canagliflozin: a pooled analysis of clinical studies. Can J Diabetes. 2016;40(3):247-57.

63. Wilding JP, Blonde L, Leiter LA, et al. Efficacy and safety of canagliflozin by baseline $\mathrm{HbA}_{\mathrm{lc}}$ and known duration of type 2 diabetes mellitus. J Diabetes Complications. 2015;29(3):438-44.

64. Blonde L, Woo V, Mathieu C, et al. Achievement of treatment goals with canagliflozin in patients with type 2 diabetes mellitus: a pooled analysis of randomized controlled trials. Curr Med Res Opin. 2015 Nov;31(11):1993-2000.

65. Bailey RA, Vijapurkar U, Meininger G, et al. Diabetes-related composite quality end point attainment: canagliflozin versus sitagliptin based on a pooled analysis of 2 clinical trials. Clin Ther. 2015;37(5):1045-54.

66. Leiter LA, Langslet G, Vijapurkar U, et al. Simultaneous reduction in both HbA1c and body weight with canagliflozin versus glimepiride in patients with type 2 diabetes on metformin. Diabetes Ther. 2016;7(2):269-78. 67. Schernthaner G, Lavalle-Gonzalez FJ, Davidson JA, et al. Canagliflozin provides greater attainment of both HbA1c and body weight reduction versus sitagliptin in patients with type 2 diabetes. Postgrad Med. 2016;128(8):725-30.

68. Leiter LA, Forst T, Polidori D, et al. Effect of canagliflozin on liver function tests in patients with type 2 diabetes. Diabetes Metab. 2016;42(1):25-32.

69. Buysman EK, Chow W, Henk HJ, et al. Characteristics and outcomes of patients with type 2 diabetes mellitus treated with canagliflozin: a real-world analysis. BMC Endo Dis. 2015 Nov 02;15:67.

70. Chow W, Miyasato G, Kokkotos FK, et al. Real-world canagliflozin utilization: glycemic control among patients with type 2 diabetes mellitus - a multi-database synthesis. Clin Ther. 2016;38(9):2071-82.

71. Bailey RA, Schwab P, Xu Y, et al. Glycemic control outcomes after canagliflozin initiation: observations in a Medicare and commercial managed care population in clinical practice. Clin Ther. 2016;38(9):2046-57.e2.

72. Usiskin K, Kline I, Fung A, et al. Safety and tolerability of canagliflozin in patients with type 2 diabetes mellitus: pooled analysis of phase 3 study results. Postgrad Med. 2014 May;126(3):16-34.

73. Erondu N, Desai M, Ways K, et al. Diabetic ketoacidosis and related events in the canagliflozin type 2 diabetes clinical program. Diabetes Care. 2015 Sep;38(9):1680-6. 
74. Yang XP, Lai D, Zhong XY, et al. Efficacy and safety of canagliflozin in subjects with type 2 diabetes: systematic review and meta-analysis. Eur J Clin Pharmacol. 2014 Oct;70(10):1149-58.

75. Nyirjesy P, Sobel JD, Fung A, et al. Genital mycotic infections with canagliflozin, a sodium glucose co-transporter 2 inhibitor, in patients with type 2 diabetes mellitus: a pooled analysis of clinical studies. Curr Med Res Opin. 2014 Feb 21;30(6):1109-19.

76. Nicolle LE, Capuano G, Fung A, et al. Urinary tract infection in randomized phase III studies of canagliflozin, a sodium glucose co-transporter 2 inhibitor. Postgrad Med. 2014 Jan;126(1):7-17.

77. Fioretto P, Weir M, Gilbert R, et al. Effect of longer-term canagliflozin treatment on eGFR in patients with type 2 diabetes mellitus and various degrees of baseline renal function [abstract no. 747]. Diabetologia. 2015;58(Suppl 1):S358-9

78. Desai M, Yavin Y, Balis D, et al. Renal Safety of Canagliflozin, a Sodium Glucose Co-transporter 2 Inhibitor, in Patients With Type 2 Diabetes Mellitus. Diabetes Obes Metab. 2017;12:12.

79. Janssen Pharmaceuticals Inc. Important safety information. Interim safety analysis from an ongoing trial observed a higher incidence of lower limb amputations (primarily of the toe) in patients treated with Invokana ${ }^{\circledR}$ (canagliflozin). Reminder regarding the importance of foot care in patients with diabetes [media release]. 20 May 2016. https://www.janssenmd.com/sites/default/files/pdf/CAN_DHCP_Letter_2016-05-20.pdf.

80. Watts NB, Bilezikian JP, Usiskin K, et al. Effects of canagliflozin on fracture risk in patients with type 2 diabetes mellitus. J Clin Endocrinol Metab. 2016 Jan;101(1):157-66.

81. Rosenthal N, Meininger G, Ways K, et al. Canagliflozin: a sodium glucose co-transporter 2 inhibitor for the treatment of type 2 diabetes mellitus. Ann N Y Acad Sci. 2015;1358:28-43.

82. Cefalu WT, Riddle MC. SGLT2 inhibitors: the latest "new kids on the block"! Diabetes Care. 2015 Mar;38(3):3524.

83. NICE. Canagliflozin, dapagliflozin and empagliflozin as monotherapies for treating type 2 diabetes. 2016. https://www.nice.org.uk. Accessed 8 May 2017.

84. American Heart Association. Cardiovascular disease \& diabetes. 2017. http://www.heart.org. Accessed 8 May 2017. 85. Van Gaal L, Scheen A. Weight management in type 2 diabetes: current and emerging approaches to treatment. Diabetes Care. 2015 Jun;38(6):1161-72.

86. Majewski C, Bakris GL. Blood pressure reduction: an added benefit of sodium-glucose cotransporter 2 inhibitors in patients with type 2 diabetes. Diabetes Care. 2015 Mar;38(3):429-30.

87. DeFronzo RA, Norton L, Abdul-Ghani M. Renal, metabolic and cardiovascular considerations of SGLT2 inhibition. Nature reviews Nephrology. 2017 Jan;13(1):11-26.

88. Whittington C, Schubert A, Neslusan C. An assessment of the relative efficacy of sodium glucose co-transporter 2 inhibitors as add-on to metformin in patients with type 2 diabetes mellitus [abstract no. PDB5]. Value Health.

2016;19:A665

89. Taieb V, Pacou M, Schroeder M, et al. Network meta-analysis (NMA) to assess relative efficacy measured as percentage of patients treated to HbA1c target with canagliflozin in patients with type 2 diabetes mellitus (T2DM) inadequately controlled on metformin and sulphonylurea (MET+SU) [abstract no. PDB5]. Value Health. 2015;18(7):A598

90. Schroeder M, Taieb V, Belhadi D, et al. Bayesian network meta-analysis (NMA) to assess the relative efficacy of canagliflozin monotherapy over 26 weeks in patients with type 2 diabetes mellitus (T2DM) [abstract no. PDB22]. Value Health. 2015;18(3):A56

91. Van Sanden S, Diels J, Guillon P, et al. Bayesian network meta-analysis (NMA) to assess relative efficacy of canagliflozin (CANA) versus glucagon-like peptide-1 (GLP-1) agonists in dual and triple therapy in patients with type 2 diabetes mellitus (T2DM) [abstract no. PDB12]. Value Health. 2015;18(3):A54

92. Taieb V, Pacou M, Schroeder M, et al. Bayesian network meta-analysis (NMA) to assess the relative efficacy of canagliflozin in patients with type 2 diabetes mellitus (T2DM) inadequately controlled with insulin [abstract no. PDB7]. Value Health. 2015;18(7):A598

93. Schroeder M, Johansen P, Willis M, et al. The cost-effectiveness of canagliflozin versus sulphonylurea in patients with type 2 diabetes with inadequate control on metformin monotherapy in the UK [abstract no. P573]. Diabet Med. 2015;32(Suppl 1):205 
94. Schroeder M, Johansen P, Willis M, et al. The cost-effectiveness of canagliflozin (CANA) versus dapagliflozin (DAPA) $10 \mathrm{mg}$ and empagliflozin (EMPA) $25 \mathrm{mg}$ in patients with type 2 diabetes mellitus (T2DM) as monotherapy in the United Kingdom [abstract no. PDB59]. Value Health. 2015;18(7):A607

95. Nielsen AT, Pitcher A, Lovato E, et al. The cost-effectiveness evaluation of canagliflozin versus dapagliflozin in patients with type 2 diabetes mellitus inadequately controlled on metformin monotherapy in Spain [abstract no.

PDB50]. Value Health. 2015;18(3):A61

96. Nielsen AT, Pitcher A, Lovato E, et al. The cost-effectiveness of canagliflozin (CANA) versus sitagliptin (SITA) as an add-on to metformin or metformin plus sulphonylurea in the treatment of type 2 diabetes mellitus in Spain [abstract no. PDB55]. Value Health. 2015;18(3):A62

97. Evans M, Schroeder M, Schubert A, et al. The cost of glycaemic target achievement with sodium glucose cotransporter 2 (SGLT 2) inhibitors in patients with type 2 diabetes mellitus (T2DM) inadequately controlled on metformin and sulphonylure (MET+SU) in the UK [abstract no. PDB24]. Value Health. 2016;19:A669.

98. Ravasio R, Pisarra P, Comaschi M. Cost-minimization analysis (CMA) of canagliflozin compared to glimepiride and sitagliptin as dual therapy in combination with metformin [abstract no. PDB72]. Value Health. 2015;18(7):A610 
Table 1 Efficacy of oral canagliflozin as first-line monotherapy in adults with inadequately-controlled T2D in 26-week double-blind phase 3 trials and a 26-week double-blind extension; changes from baseline are least squares means and baseline values are means

\begin{tabular}{|c|c|c|c|c|c|c|}
\hline \multirow[t]{2}{*}{ Study } & \multirow{2}{*}{$\begin{array}{l}\text { Regimen (mg od) } \\
\text { [no. of pts] }\end{array}$} & \multirow{2}{*}{$\begin{array}{l}\text { Week } \\
\text { of eval }\end{array}$} & \multicolumn{2}{|c|}{ Change from BL $[\mathrm{BL}]$} & \multirow{2}{*}{$\begin{array}{l}\% \text { pts with } \\
\mathrm{HbA}_{1 \mathrm{C}}<7 \%\end{array}$} & \multirow{2}{*}{$\begin{array}{l}\text { Change from BL } \\
{[\mathrm{BL}]} \\
\text { Bodyweight }(\mathrm{kg})\end{array}$} \\
\hline & & & $\mathrm{HbA}_{1 \mathrm{C}} \mathrm{a}(\%)$ & $\mathrm{FPG}(\mathrm{mmol} / \mathrm{L})$ & & \\
\hline Stenlöf et al [13] & CAN 100 [195] & 26 & $-0.77 *[8.1]$ & $-1.5 *[9.6]$ & $44.5^{*}$ & $-2.5 *[85.9]$ \\
\hline \multirow[t]{2}{*}{ (CANTATA-M) } & CAN 300 [197] & & $-1.03 *[8.0]$ & $-1.9 *[9.6]$ & $62.4 *$ & $-3.4 *[86.9]$ \\
\hline & PL [192] & & $+0.14[8.0]$ & $+0.5[9.2]$ & 20.6 & $-0.5[87.5]$ \\
\hline Stenlöf et al [33] & CAN 100 [166] & 52 & $-0.81[8.0]$ & $-1.5[9.5]$ & 52.4 & $-2.8[86.4]$ \\
\hline$(\text { CANTATA-M ext })^{b}$ & CAN 300 [166] & & $-1.11[8.0]$ & $-2.2[9.4]$ & 64.5 & $-3.9[87.2]$ \\
\hline \multirow[t]{3}{*}{ Jodar et al [27] } & CAN $100\left[712^{c}\right]$ & 26 & $-1.37^{\mathrm{d}}\left[8.8^{\mathrm{c}}\right]$ & NR & 38.8 & NR \\
\hline & CAN 300 & & $-1.42^{\mathrm{d}}$ & NR & 42.8 & NR \\
\hline & MET-XR & & -1.30 & NR & 43.0 & NR \\
\hline
\end{tabular}

$B L$ baseline, $C A N$ canagliflozin, ext extension, eval evaluation, $F P G$ fasting plasma glucose, $H b A_{I C}$ glycosylated haemoglobin, $M E T$-XR metforminextended release, $N R$ not reported, od once daily, $P L$ placebo, $p t s$ patients

$* p<0.001$ vs. PL

${ }^{a}$ Primary endpoint at week 26

${ }^{\mathrm{b}}$ Pts $(n=155)$ originally randomized to PL in CANTATA-M were switched to sitagliptin $100 \mathrm{mg}$ od for this ext; efficacy data were not reported

${ }^{\mathrm{c}}$ Value is for the whole trial population

${ }^{\mathrm{d}}$ Noninferiority of CAN 100 or $300 \mathrm{mg}$ od vs. MET-XR was established (no further details reported) 
Drugs (2017)

DOI

Table 2 Efficacy of oral canagliflozin as add-on therapy to metformin, with or without other oral antihyperglycaemic agents, in adults with inadequately-controlled T2D in randomized, double-blind, phase 3 (or phase 4 [37]) trials and a double-blind extension

\begin{tabular}{|c|c|c|c|c|c|c|}
\hline \multirow[t]{2}{*}{ Study (acronym) } & \multirow[t]{2}{*}{ Regimen (mg od) [no. of pts] } & \multirow{2}{*}{$\begin{array}{l}\text { Week } \\
\text { of eval }\end{array}$} & \multicolumn{2}{|c|}{ Change from BL [BL] } & \multirow{2}{*}{$\begin{array}{l}\% \text { of pts } \\
\text { with } \\
\mathrm{HbA}_{1 \mathrm{C}} \\
<7 \%\end{array}$} & \multirow{2}{*}{$\begin{array}{l}\text { Change from } \mathrm{BL} \\
{[\mathrm{BL}]} \\
\text { Bodyweight }(\mathrm{kg})\end{array}$} \\
\hline & & & $\mathrm{HbA}_{1 \mathrm{C}}{ }^{\mathrm{a}}(\%)$ & FPG (mmol/L) & & \\
\hline \multicolumn{7}{|l|}{ Add-on to MET } \\
\hline Lavalle-González et al [34] & CAN 100 + MET [368] & 26 & $-0.79 * *[7.9]$ & $-1.5 * *[9.4]$ & $46^{* *}$ & $-3.3 * *[88.7]$ \\
\hline \multirow[t]{6}{*}{ (CANTATA-D) } & CAN $300+$ MET [367] & & $-0.94 * *[8.0]$ & $-2.1 * *[9.6]$ & $58 * *$ & $-3.6^{* *}[85.4]$ \\
\hline & SIT $100+$ MET [366] & & $-0.82^{\mathrm{b}}[7.9]$ & $-1.1^{\mathrm{b}}[9.4]$ & $55^{\mathrm{b}}$ & $-1.1^{\mathrm{b}}[87.6]$ \\
\hline & PL + MET [183] & & $-0.17[8.0]$ & $+0.1[9.1]$ & 30 & $-1.1[86.7]$ \\
\hline & CAN $100+$ MET [368] & 52 & $-0.73^{\mathrm{c}}[7.9]$ & $-1.5 \dagger[9.4]$ & $41^{\mathrm{d}}$ & $-3.3 \dagger[88.7]$ \\
\hline & CAN 300 + MET [367] & & $-0.88^{\mathrm{c}}[8.0]$ & $-2.0 \dagger[9.6]$ & $55^{\mathrm{d}}$ & $-3.7 \dagger[85.4]$ \\
\hline & SIT 100 + MET [366] & & $-0.73[7.9]$ & $-1.0[9.4]$ & 51 & $-1.2[87.6]$ \\
\hline Cefalu et al [35] & CAN 100 + MET [483] & 52 & $-0.82^{\mathrm{c}}[7.8]$ & $-1.35^{\mathrm{b}}[9.2]$ & $54^{\mathrm{b}}$ & $-3.7 \dagger \dagger^{\mathrm{e}}[86.8]$ \\
\hline \multirow[t]{2}{*}{ (CANTATA-SU) } & CAN 300 + MET [485] & & $-0.93^{\mathrm{c}}[7.8]$ & $-1.52^{\mathrm{b}}[9.1]$ & $60^{\mathrm{b}}$ & $-4.0+\dagger^{\mathrm{e}}[86.6]$ \\
\hline & GLIM + MET [482] & & $-0.81[7.8]$ & $-1.02[9.2]$ & 56 & $+0.7[86.6]$ \\
\hline Leiter et al [36] & CAN 100 + MET [393] & 104 & $-0.65[7.8]$ & $-1.1^{\mathrm{f}}[9.2]$ & $43^{\mathrm{b}}$ & $-4.1^{\mathrm{f}}[86.8]$ \\
\hline \multirow[t]{2}{*}{ (CANTATA-SU ext) } & CAN 300 + MET [377] & & $-0.74^{\mathrm{f}}[7.8]$ & $-1.3^{\mathrm{f}}[9.1]$ & $50^{\mathrm{b}}$ & $-4.2^{\mathrm{f}}[86.6]$ \\
\hline & GLIM + MET [381] & & $-0.55[7.8]$ & $-0.6[9.2]$ & 44 & $+0.9[86.6]$ \\
\hline \multicolumn{7}{|c|}{ Add-on to MET + Other Oral Antihyperglycaemic Agent } \\
\hline Wilding et al [14] & CAN $100+$ MET + SU [157] & 26 & $-0.85 * *[8.1]$ & $-1.0 * *[9.6]$ & $43 * *$ & $-1.9 * * \mathrm{e}[93.5]$ \\
\hline \multirow{5}{*}{ (CANTATA-MSU) } & CAN $300+$ MET + SU [156] & & $-1.06^{* *}[8.1]$ & $-1.7 * *[9.3]$ & $57 * *$ & $-2.5^{* * \mathrm{e}}[93.5]$ \\
\hline & $\mathrm{PL}+\mathrm{MET}+\mathrm{SU}[156]$ & & $-0.13[8.1]$ & $+0.2[9.4]$ & 18 & $-0.8[90.8]$ \\
\hline & CAN $100+$ MET + SU [157] & $52^{\mathrm{b}}$ & $-0.74[8.1]$ & $-1.1[9.6]$ & 39 & $-2.0[93.5]$ \\
\hline & CAN $300+$ MET + SU [156] & & $-0.96[8.1]$ & $-1.5[9.3]$ & 53 & $-3.1[93.5]$ \\
\hline & $\mathrm{PL}+\mathrm{MET}+\mathrm{SU}[156]$ & & $+0.01[8.1]$ & $+0.6[9.4]$ & 19 & $-1.0[90.8]$ \\
\hline Schernthaner et al [15] & CAN $300+\mathrm{MET}+\mathrm{SU}$ [377] & 52 & $-1.03^{\mathrm{c}}[8.1]$ & $-1.7 * *[9.4]$ & $48^{b}$ & $-2.3 * *[87.6]$ \\
\hline (CANTATA-D2) & SIT $100+$ MET + SU [378] & & $-0.66[8.1]$ & $-0.3[9.1]$ & 35 & $+0.1[89.6]$ \\
\hline \multirow[t]{5}{*}{ Forst et al [16] } & CAN $100+$ MET + PIO [113] & 26 & $-0.89 * *[8.0]$ & $-1.5 * *[9.4]$ & $47 *$ & $-2.6^{* *}[94.2]$ \\
\hline & CAN $300+$ MET + PIO [114] & & $-1.03 * *[7.8]$ & $-1.8 * *[9.1]$ & $64 * *$ & $-3.7 * *[94.4]$ \\
\hline & $\mathrm{PL}+\mathrm{MET}+\mathrm{PIO}^{\mathrm{g}}[115]$ & & $-0.26[8.0]$ & $+0.1[9.1]$ & 33 & $-0.2[94.0]$ \\
\hline & CAN $100+$ MET + PIO [103] & 52 & $-0.92[8.0]$ & $-1.5[9.4]$ & 52 & $-2.5[94.2]$ \\
\hline & CAN $300+$ MET + PIO [96] & & $-1.03[7.8]$ & $-1.8[9.1]$ & 66 & $-3.6[94.4]$ \\
\hline Rodbard et al [37] & $\mathrm{CAN}^{\mathrm{h}}+\mathrm{MET}+\operatorname{SIT}[107]$ & 26 & $-0.91 * *[8.5]$ & $-1.7 * *[10.3]$ & $32 * *$ & $-3.1 * * \mathrm{e}[93.8]$ \\
\hline & $\mathrm{PL}+\mathrm{MET}+\mathrm{SIT}[106]$ & & $-0.01[8.4]$ & $-0.1[10.0]$ & 12 & $-1.6[89.9]$ \\
\hline
\end{tabular}

Changes from $\mathrm{BL}$ are least squares means and $\mathrm{BL}$ values are means

$B L$ baseline, $B G D$ between-group difference, $C A N$ canagliflozin, ext extension, eval evaluation, $F P G$ fasting plasma glucose, GLIM glimepiride (uptitrated to 6 or $8 \mathrm{mg}$ od), $H b A_{l C}$ glycosylated haemoglobin, $M E T$ metformin (generally $\geq 2000 \mathrm{mg} / \mathrm{day} ; \geq 1500 \mathrm{mg} /$ day if higher dosages not tolerated, $o d$ once daily, $P I O$ pioglitazone ( 30 or $45 \mathrm{mg} /$ day), $P L$ placebo, pts patients, SIT sitagliptin, $S U$ sulfonylurea ( $\geq 50 \%$ maximal dosage)

$* p<0.01, * * p \leq 0.001$ vs. PL; $\dagger p<0.001$, $\dagger \dagger p<0.0001$ vs. active comparator group

${ }^{a}$ Primary endpoint at week $26[14, \underline{16}, \underline{34}, \underline{37]}$ or $52[15, \underline{35}]$

${ }^{\mathrm{b}}$ No formal statistics performed/reported for CAN vs. PL [14], GLIM [35, 36] or SIT [15], or SIT vs. PL [34], for these endpoints/timepoints

${ }^{c}$ Based on prespecified criteria, CAN 100 was noninferior to, and CAN 300 more effective than, SIT [15, 34] and GLIM [35]

${ }^{\mathrm{d}}$ Difference vs. SIT was not significant for CAN 300 but significant for CAN 100, based on the $95 \%$ CIs of the odds ratios

${ }^{\mathrm{e}} \mathrm{P}$-values are for percentage changes in bodyweight, and are assumed to also apply to kg changes

${ }^{\mathrm{f}}$ Significantly favoured CAN vs. GLIM, based on $95 \%$ CI for BGD

${ }^{g}$ Ninety pts in this group entered the 26 -week ext, during which they received SIT + MET + PIO to maintain blinding

${ }^{\mathrm{h}}$ Pts received CAN 100 or $300 \mathrm{mg}$ od; pooled data for these groups are presented 
Table 3 Efficacy of oral canagliflozin as add-on therapy to insulin- or incretin mimetic-based therapy or sulfonylurea monotherapy in prespecified [ $\underline{43}, \underline{44}]$ or post hoc [ㄷ] subgroup analyses of the phase 3 CANVAS trial

\begin{tabular}{|c|c|c|c|c|c|c|c|c|}
\hline \multirow[t]{2}{*}{ Regimen (mg od) [no. of pts] } & \multirow{2}{*}{$\begin{array}{l}\text { Week } \\
\text { of } \\
\text { eval }\end{array}$} & \multicolumn{2}{|l|}{$\mathrm{HbA}_{1 \mathrm{C}}(\%)$} & \multicolumn{2}{|c|}{ FPG (mmol/L) } & \multirow{2}{*}{$\begin{array}{l}\% \text { of pts } \\
\text { with } \\
\mathrm{HbA}_{1 \mathrm{C}} \\
<7 \%\end{array}$} & \multicolumn{2}{|c|}{ Bodyweight (kg) } \\
\hline & & $\begin{array}{l}\text { Change from } \\
\text { BL [BL] }\end{array}$ & $\begin{array}{l}\text { Diff vs. } \\
\text { PL }\end{array}$ & $\begin{array}{l}\text { Change } \\
\text { from BL }\end{array}$ & $\begin{array}{l}\text { Diff vs. } \\
\text { PL }\end{array}$ & & $\begin{array}{l}\text { Change } \\
\text { from BL }\end{array}$ & $\begin{array}{l}\text { Diff vs. } \\
\text { PL }\end{array}$ \\
\hline \multicolumn{9}{|l|}{ Add-on to INS therapy [43] } \\
\hline CAN $100+$ INS \pm OAA $[661]$ & 18 & $-0.63^{\mathrm{a}}[8.3]$ & $-0.62 * *$ & NR [9.2] & $-1.2 * *$ & $19.8 * *$ & NR [94.4] & $-1.9 * *$ \\
\hline CAN $300+$ INS \pm OAA [660] & & $-0.75^{\mathrm{a}}[8.3]$ & $-0.73 * *$ & NR [9.2] & $-1.6 * *$ & $25.8 * *$ & NR [94.8] & $-2.4 * *$ \\
\hline $\mathrm{PL}+\mathrm{INS} \pm \mathrm{OAA}[636]$ & & $-0.1^{\mathrm{a}}[8.3]$ & & $\mathrm{NR}[9.2]$ & & 8.3 & NR [94.8] & \\
\hline CAN $100+$ INS \pm OAA [664] & 52 & $-0.55[8.3]$ & $-0.58^{\mathrm{b}}$ & NR [9.2] & $-1.1^{\mathrm{b}}$ & 23.2 & NR [94.4] & $-2.8^{\mathrm{b}}$ \\
\hline CAN $300+$ INS \pm OAA [664] & & $-0.69[8.3]$ & $-0.73^{\mathrm{b}}$ & NR [9.2] & $-1.5^{\mathrm{b}}$ & 28.6 & NR [94.8] & $-3.5^{\mathrm{b}}$ \\
\hline $\mathrm{PL}+\mathrm{INS} \pm$ OAA [639] & & $+0.03[8.3]$ & & NR [9.2] & & 9.9 & NR [94.8] & \\
\hline \multicolumn{9}{|l|}{ Add-on to SU [44] } \\
\hline CAN $100+$ SU [42] & 18 & $-0.70[8.3]$ & $-0.74 * *$ & $-1.4[10.3]$ & -2.1 & 25 & $-0.6[85.1]$ & $-0.4 \%$ \\
\hline CAN $300+$ SU [40] & & $-0.79[8.3]$ & $-0.83 * *$ & $-2.0[9.8]$ & $-2.7 * *$ & 33 & $-2.0[80.4]$ & $-1.8 \% *$ \\
\hline $\mathrm{PL}+\mathrm{SU}[45]$ & & $+0.04[8.5]$ & & $+0.7[10.3]$ & & 5 & $-0.2[85.5]$ & \\
\hline \multicolumn{9}{|c|}{ Add-on to incretin-mimetic therapy [45] } \\
\hline CAN 100 + DPP4i \pm OAA [103] & 18 & $-0.46[8.1]$ & $-0.56^{\mathrm{b}}$ & NR & $-1.1^{\mathrm{b}}$ & 22 & $-2.7[91.5]$ & $-2.0^{\mathrm{b}}$ \\
\hline CAN $300+$ DPP $4 \mathrm{i} \pm$ OAA [111] & & $-0.64[8.0]$ & $-0.75^{\mathrm{b}}$ & NR & $-1.5^{\mathrm{b}}$ & $34^{\mathrm{b}}$ & $-3.5[92.4]$ & $-2.7^{\mathrm{b}}$ \\
\hline $\mathrm{PL}+\mathrm{DPP} 4 \mathrm{i} \pm \mathrm{OAA}[102]$ & & $+0.1[8.1]$ & & NR & & 15 & $-0.8[88.6]$ & \\
\hline CAN 100 + GLP-1ra \pm OAA [35] & 18 & $-0.83[8.2]$ & $-1.00^{\mathrm{b}}$ & NR & $-1.8^{\mathrm{b}}$ & $29^{\mathrm{b}}$ & $-3.3[109]$ & $-2.7^{\mathrm{b}}$ \\
\hline CAN 300 + GLP-1ra \pm OAA [30] & & $-0.89[8.3]$ & $-1.06^{\mathrm{b}}$ & NR & $-2.5^{\mathrm{b}}$ & $35^{\mathrm{b}}$ & $-3.9[111]$ & $-3.3^{\mathrm{b}}$ \\
\hline $\mathrm{PL}+\mathrm{GLP}-1 \mathrm{ra} \pm$ OAA [30] & & $+0.17[7.9]$ & & NR & & 7 & $-0.6[106]$ & \\
\hline \multicolumn{9}{|c|}{$\begin{array}{l}\text { Changes from BL are least squares means, BL values are means. INS dosage was } \geq 20 \text { IU/day; SU, DPP4i and GLP-1ra dosages were stable } \\
B L \text { baseline, } C A N \text { canagliflozin, diff difference, } D P P 4 i \text { dipeptidyl peptidase } 4 \text { inhibitor, } e v a l \text { evaluation, } F P G \text { fasting plasma glucose, GLP-1ra } \\
\text { glucagon-like peptide- } 1 \text { receptor agonist, } H b A_{I C} \text { glycosylated haemoglobin, } I N S \text { insulin, } N R \text { not reported, } O A A \text { other antihyperglycaemic agent, } o d \\
\text { once daily, } P L \text { placebo, } p t s \text { patients, } S U \text { sulfonylurea } \\
* p<0.02, * * p<0.001 \text { vs. PL }\end{array}$} \\
\hline${ }^{a}$ Estimated from a graph & & & & & & & & \\
\hline
\end{tabular}

